\title{
Dirk Fox
}

\section{Elektronische Gesundheitskarte}

\section{Hintergrund}

Ursprünglich sollte die elektronische Gesundheitskarte (kurz: eGK) die 1995 eingeführte Krankenversicherungskarte (kurz: KVK), eine reine Speicherchipkarte, bereits zum 01.01.2006 ablösen $(\$ 291 \mathrm{a}$ SGB V). ${ }^{1}$ Neben einem verbesserten $\mathrm{Zu}$ griffsschutz auf die auf der Karte gespeicherten Daten ist eine Kernzielsetzung der eGK die Verhinderung des zunehmenden Missbrauchs von Krankenversichertenkarten zur Erschleichung medizinischer Leistungen. Dies soll durch eine eindeutige Zuordnung der Karte zum Versicherten mittels der Aufbringung eines Fotos und der Ausgestaltung der Karte als elektronischer Ausweis erreicht werden.

Darüber hinaus soll die eGK geeignet sein, zusätzliche Funktionen wie die Speicherung ärztlicher Verordnungen (elektronisches Rezept) und die europäische Krankenversichertenkarte zu speichern, sowie freiwillige Zusatzanwendungen wie z. B. Notfallversorgungsdaten, die elektronische Patientenakte oder persönliche Arzneimittel-Unverträglichkeiten. Sie soll zudem die Abrechnung von Leistungen im Gesundheitswesen durch die Vermeidung von Medienbrüchen vereinfachen.

\section{Technik}

Die eGK ist eine Prozessor-Chipkarte. Auf ihr sind zunächst einmal die auch auf der KVK gespeicherten Versichertendaten abgelegt:

- Die Bezeichnung der Krankenkasse zzgl. eines Kennzeichens für die zuständige Kassenärztliche Vereinigung,

- Name und Vorname des Versicherten,

- Geburtsdatum, Geschlecht, Anschrift und Krankenversichertennummer,

- Versicherten- und Zuzahlungsstatus,

- Beginn des Versicherungsschutzes,

- Ablauf der Gültigkeit der Karte.

Zur Missbrauchsvermeidung wird die Karte mit einem Lichtbild des Versicherten versehen.

1 Siehe Bizer, Gesundheitskarte, Gateway, DuD 4/2004, S. 243
Darüber hinaus können auf der mit 32 kByte Speicher ausgestatteten Chipkarte bis zu acht elektronische Rezepte und die Notfallversorgungsdaten gespeichert werden. Alle weiteren Daten, wie z. B. die elektronische Patientenakte, sollen in der Telematik-Infrastruktur auf zentralen Servern gespeichert werden.

\section{Datenschutz}

Neben geeigneten Maßnahmen zur Erfüllung der Auskunftsrechte der Betroffenen über Inhalt und Herkunft der gespeicherten Daten ( $\$ \$ 19,34$ BDSG) und des Berichtigungs- und Löschanspruchs bezüglich falscher oder unzulässig gespeicherter Daten ( $\$ \$ 20,35$ BDSG) müssen die auf der eGK gespeicherten Daten technisch vor unberechtigten (insbesondere also nicht erforderlichen) Zugriffen und Verfälschung geschützt werden.

Daraus leiten sich komplexe Berechtigungsmodelle $\mathrm{ab}$, in denen zahlreiche Rollen (Arzt, Apotheker, Nothelfer etc.) mit verschiedenen Rechten unterschieden werden. Um diese Zugriffsbeschränkungen technisch umzusetzen, müssen sich die Rolleninhaber vor Freigabe des Zugriffs ihrerseits elektronisch mit ihrem Heilberufsausweis und qualifizierter elektronischer Signatur ausweisen, und der Versicherte muss seinerseits durch PINEingabe der Datenfreigabe (ausgenommen bei einem Zugriff auf die Notfallversorgungsdaten) zustimmen.

Zur Nachvollziehbarkeit und Datenschutzkontrolle werden mindestens die letzten 50 Zugriffe auf die Daten protokolliert; diese Protokolldaten sind ihrerseits wiederum vor missbräuchlicher Nutzung zu schützen.

\section{Einführung}

Die Kosten für den Aufbau der für die Nutzung der eGK erforderlichen Telematik-Infrastruktur werden auf bis zu 1,4 Mrd. € geschätzt. Die Einführung der eGK soll(te) durch das 2003 vom Gesundheitsministerium ausgeschriebene Projekt „better IT for better health“ (bIT4health) vorbereitet und begleitet werden.

Anfang 2005 gründeten die Spitzenorganisationen des deutschen Gesundheitswesens (Leistungserbringer wie Ärzte- und Apothekerorganisationen und Kostenträger wie die gesetzlichen und privaten Krankenversicherungen) für die Einführung, Pflege und Weiterentwicklung der eGK die Gesellschaft für Telematikanwendungen der Gesundheitskarte $\mathrm{mbH}$ (gematik). Sie ist zuständig für

- die technische Spezifikation der Dienste, Datenformate und Komponenten der für die eGK erforderliche Telematik-Infrastruktur,

- den Test und die Zulassung von Diensten und Komponenten, die in der Infrastruktur eingesetzt werden, und

- den Betrieb (eines Teils) der Telematikinfrastruktur.

Tatsächlich gelang die zum 01.01.2006 geplante flächendeckende Einführung der eGK nicht. Im Oktober 2006 wurde - auch vor dem Hintergrund anhaltender Kritik am unzureichenden Schutz der Gesundheitsdaten ${ }^{2}$ und Mängeln in den Softwareprototypen - ein vierstufiges Testverfahren verordnet, das vor der Einführung den Einsatz der eGK in Testregionen vorsieht. Im Oktober 2009 begann die Einführung der eGK in der Pilotregion Nordrhein, nachdem zuvor der Verband der privaten Krankenversicherungen im Juli 2009 seine Teilnahme am Roll-Out der eGK aus Zweifeln an der Investitionssicherheit abgesagt hatte.

Schließlich stoppte der (neue) Bundesgesundheitsminister aufgrund von Zweifeln an der technischen Sicherheit die Funktionen elektronisches Rezept und elektronische Patientenakte.

Mit dem am 12.11.2010 vom Bundestag verabschiedeten GKV-Finanzierungsgesetz sollen die Krankenversicherungen nun verpflichtet werden, bis Ende 2011 wenigstens $10 \%$ ihrer Versicherten mit einer elektronischen Gesundheitskarte auszustatten.

2 Siehe u.a. Weichert, Die elektronische Gesundheitskarte, DuD 7/2004, S. 391-403; Schwerpunktheft Telematik im Gesundheitswesen, DuD 3/2006. 